\title{
ON COMMON FIXED POINTS OF PAIRS OF A SINGLE AND A MULTIVALUED COINCIDENTALLY COMMUTING MAPPINGS IN $D$-METRIC SPACES
}

\author{
B. C. DHAGE, A. JENNIFER ASHA, and S. M. KANG \\ Received 10 December 2002 and in revised form 15 February 2003
}

\begin{abstract}
The present paper studies some common fixed-point theorems for pairs of a single-valued and a multivalued coincidentally commuting mappings in $D$-metric spaces satisfying a certain generalized contraction condition. Our result generalizes more than a dozen known fixed-point theorems in $D$-metric spaces including those of Dhage (2000) and Rhoades (1996).
\end{abstract}

2000 Mathematics Subject Classification: 47H10, 54H25.

1. Introduction. The concept of a $D$-metric space introduced by the first author in [1] is as follows. A nonempty set, together with a function $\rho: X \times X \times$ $X \rightarrow[0, \infty)$, is called a $D$-metric space and denoted by $(X, \rho)$ if the function $\rho$, called a $D$-metric on $X$, satisfies the following properties:

(i) $\rho(x, y, z)=0 \Leftrightarrow x=y=z$ (coincidence),

(ii) $\rho(x, y, z)=0=\rho(p\{x, y, z\})$ (symmetry), where $p$ is a permutation,

(iii) $\rho(x, y, z) \leq \rho(x, y, a)+\rho(x, a, z)+\rho(a, y, z)$ for all $x, y, z, a \in X$ (tetrahedral inequality).

It is known that the $D$-metric $\rho$ in a continuous function on $X^{3}$ in the topology of $D$-metric convergence is Hausdorff. The details of a $D$-metric space and its topological properties appear in Dhage [8]. Some specific examples of a $D$-metric space are presented in Dhage [2].

A sequence $\left\{x_{n}\right\} \subset X$ is called convergent and converges to a point $x$ if $\lim _{m, n} \rho\left(x_{m}, x_{n}, x\right)=0$. Again a sequence $\left\{x_{n}\right\} \subset X$ is called $D$-Cauchy if $\lim _{m, n, p} \rho\left(x_{m}, x_{n}, x_{p}\right)=0$. A complete $D$-metric space $X$ is one in which every $D$-Cauchy sequence converges to a point in $X$. A subset $S$ of a $D$-metric space $X$ is called bounded if there exists a constant $k>0$ such that $\rho(x, y, z) \leq k$ for all $x, y, z \in X$ and the constant $k$ is called a $D$-bound of $S$. The smallest among all such $D$-bounds $k$ of $S$ is called the diameter of $X$ and it is denoted by $\delta(S)$.

Let $2^{X}$ and $\mathrm{CB}(X)$ denote the classes of nonempty closed and nonempty, closed, bounded subsets of $X$, respectively. A correspondence $F: X \rightarrow 2^{X}$ is called a multivalued mapping on a $D$-metric space $X$, and a point $u \in X$ is called a fixed point of $F$ if $u \in F u$. 
In [3], the first author has defined a notion of the generalized or Kasusai $D$-metric on $X$. Let $\kappa:(\mathrm{CB}(X))^{3} \rightarrow[0, \infty)$ be a function defined by

$$
\kappa(A, B, C)=\inf \{\epsilon>0 \mid A \cup B \subset N(c, \epsilon), B \cup C \subset N(A, \epsilon), C \cup A \subset N(B, \epsilon)\},
$$

where $N(A, \epsilon)=\cup_{a \in A} N(a, \epsilon), N(a, \epsilon)=\left\{x \in N^{*}(a, \epsilon) \mid \rho(a, x, y)<\epsilon\right.$ for all $\left.y \in N^{*}(a, \epsilon)\right\}$, and $N^{*}(a, \epsilon)=\{x \in X \mid \rho(a, x, x)<\epsilon\}$.

The definition (1.1) is equivalent to

$$
\kappa(A, B, C)=\max \left\{\sup _{a \in A, b \in B} D(a, b, c), \sup _{b \in B, c \in C} D(b, c, A), \sup _{c \in C, a \in A} D(c, a, B)\right\},
$$

where $D(a, b, c)=\inf \{\rho(a, b, c) \mid c \in C\}$.

Define

$$
\begin{aligned}
D(A, B, C) & =\inf \{\rho(a, b, c) \mid a \in A, b \in B, c \in C\}, \\
\delta(A, B, C) & =\sup \{\rho(a, b, c) \mid a \in A, b \in B, c \in C\} .
\end{aligned}
$$

Notice that $D$ and $\delta$ are continuous functions on $(\mathrm{CB}(X))^{3}$ and satisfy

$$
D(A, B, C) \leq \kappa(A, B, C) \leq \delta(A, B, C)
$$

A multivalued mapping $F: X \rightarrow \mathrm{CB}(X)$ is called continuous if

$$
\lim _{m, n} \rho\left(x_{m}, x_{n}, x\right)=0 \Longrightarrow \kappa\left(F x_{m}, F x_{n}, F x\right)=0
$$

In [3], the first author has proved some fixed-point theorem for multivalued contraction mappings in D-metric spaces, and in [5] he has proved some common fixed-point theorems for coincidentally commuting single-valued mappings in $D$-metric spaces satisfying a condition of generalized contraction.

In this paper, we prove some common fixed-point theorems for a pair of singlevalued and multivalued mappings in a $D$-metric space satisfying a contraction condition more general than that given in Dhage [1, 2, 3, 4, 5, 7] and Rhoades [12]. The results of this paper are new to the fixed-point theory in $D$-metric spaces and include nearly a dozen of known fixed-point theorems as special cases (see $[1,2,3,4,5,6,7,8,9,10,12])$.

2. Preliminaries. Before going to the main results of this paper, we give some preliminaries needed in the sequel.

Let $F: X \rightarrow 2^{X}$. Then by an orbit of $F$ at a point $x \in X$ we mean a set $O_{F}(x)$ in $X$ defined by

$$
O_{F}(x)=\left\{x_{0}=x, x_{n+1} \in F x_{n}, n \geq 0\right\}
$$


An orbit $O_{F}(x)$ is called bounded if $\delta\left(O_{F}(x)\right)<\infty$, and a $D$-metric space $X$ is called F-orbitally bounded if $O_{F}(x)$ is bounded for each $x \in X$. Again an $F$ orbit $O_{F}(x)$ is called complete if every $D$-Cauchy sequence in $O_{F}(x)$ converges to a point in $X$. A $D$-metric space $X$ is said to be $F$-orbitally complete if $O_{F}(x)$ is complete for each $x \in X$. Finally, $F$ is called $F$-orbitally continuous if for any sequence $\left\{x_{n}\right\} \subseteq O_{F}(x)$, we have

$$
\lim _{m, n} \rho\left(x_{m}, x_{n}, x^{*}\right)=0 \Longrightarrow \lim _{m, n} \kappa\left(F x_{m}, F x_{n}, F x^{*}\right)=0
$$

for each $x \in X$.

Let $\Phi$ denote the class of all functions $\phi:[0, \infty) \rightarrow[0, \infty)$ satisfying the following properties:

(i) $\phi$ is continuous,

(ii) $\phi$ is nondecreasing,

(iii) $\phi(t)<t, t>0$,

(iv) $\sum_{n=1}^{\infty} \phi^{n}(t)<\infty$ for all $t \in[0, \infty)$.

The function $\phi$ is called a Lipschitz control function or Lipschitz growth function and the usual growth function is $\phi(t)=\alpha t, 0 \leq t<1$. The following lemma concerning the function $\phi$ appears in [7].

LEMmA 2.1. If $\phi \in \Phi$, then $\phi^{n}(t)=0$ for each $n \in \mathbb{N}$ and $\lim _{n} \phi^{n}(t)=0$ for each $t \in[0, \infty)$.

We need the following $D$-Cauchy principle of Dhage [7] in the sequel.

LEMмA 2.2 (D-Cauchy principle). Let $\left\{x_{n}\right\}$ be a bounded sequence in a $D$ metric space $X$ with $D$-bound $k$ satisfying, for some positive real number $r$,

$$
\rho\left(x_{n}, x_{n+1}, x_{m}\right) \leq\left[\phi^{n}\left(k^{r}\right)\right]^{1 / r}
$$

for all $m>n \in \mathbb{N}$, where $\phi:[0, \infty) \rightarrow[0, \infty)$ satisfies $\sum_{n=1}^{\infty} \phi^{n}(t)<\infty$ for each $t \in[0, \infty)$. Then $\left\{x_{n}\right\}$ is a $D$-Cauchy sequence in $X$.

Proof. The proof appears in [7], but for the sake of completeness we give the details. Let $p, t \in \mathbb{N}$ be arbitrary but fixed. Then from (2.3) it follows that

$$
\begin{aligned}
\rho\left(x_{n}, x_{n+1}, x_{n+p}\right) & \leq\left[\phi^{n}\left(k^{r}\right)\right]^{1 / r}, \\
\rho\left(x_{n}, x_{n+1}, x_{n+p+t}\right) & \leq\left[\phi^{n}\left(k^{r}\right)\right]^{1 / r},
\end{aligned}
$$

for all $n \in \mathbb{N}$.

Now by repeated application of the tetrahedral inequality, we obtain

$$
\begin{aligned}
\rho( & \left.x_{n}, x_{n+p}, x_{n+p+t}\right) \\
\leq & \rho\left(x_{n}, x_{n+1}, x_{n+p}\right)+\rho\left(x_{n}, x_{n+1}, x_{n+p+t}\right)+\rho\left(x_{n+1}, x_{n+p}, x_{n+p+t}\right) \\
\leq & \rho\left(x_{n}, x_{n+1}, x_{n+p}\right)+\rho\left(x_{n}, x_{n+1}, x_{n+p+t}\right)+\rho\left(x_{n+1}, x_{n+2}, x_{n+p}\right) \\
& +\rho\left(x_{n+1}, x_{n+2}, x_{n+p+t}\right)+\rho\left(x_{n+2}, x_{n+p}, x_{n+p+t}\right)
\end{aligned}
$$




$$
\begin{aligned}
& \leq 2\left[\phi^{n}\left(k^{r}\right)\right]^{1 / r}+2\left[\phi^{n+1}\left(k^{r}\right)\right]^{1 / r}+\rho\left(x_{n+2}, x_{n+p}, x_{n+p+t}\right) \\
& \leq 2\left\{\left[\phi^{n}\left(k^{r}\right)\right]^{1 / r}+\cdots+\left[\phi^{n+p-2}\left(k^{r}\right)\right]^{1 / r}\right\}+\rho\left(x_{n+p-1}, x_{n+p}, x_{n+p+t}\right) \\
& \leq 2 \sum_{j=n}^{n+p-1}\left[\phi^{j}\left(k^{r}\right)\right]^{1 / r} .
\end{aligned}
$$

Since $\sum_{n=1}^{\infty} \phi^{n}(t)<\infty$ for each $t \in[0, \infty)$, we have $\sum_{j=1}^{\infty}\left[\phi^{j}\left(k^{r}\right)\right]^{1 / r}<\infty$ and so $\lim _{n} \sum_{j=n}^{n+p-1}\left[\phi^{j}\left(k^{r}\right)\right]^{1 / r}=0$. Now from (2.5) it follows that

$$
\lim _{n \rightarrow \infty} \rho\left(x_{n}, x_{n+p}, x_{n+p+t}\right)=0
$$

This proves that $\left\{x_{n}\right\}$ is a $D$-Cauchy sequence in $X$ and the proof of the lemma is complete.

As a direct application of Lemma 2.2, we obtain the following result proved in [5].

LEMmA 2.3. Let $\left\{x_{n}\right\}$ be a bounded sequence in a D-metric space $X$ with $D$-bound $k$ satisfying

$$
\rho\left(x_{n}, x_{n+1}, x_{m}\right) \leq \lambda^{n} k
$$

for all $m>n \in \mathbb{N}$, where $0 \leq \lambda<1$. Then $\left\{x_{n}\right\}$ is $D$-Cauchy.

We use contractive conditions of the form

$$
a^{r} \leq \phi\left(b^{r}\right)
$$

for some positive real number $r$, where $a$ and $b$ are nonnegative real numbers and $\phi \in \Phi$, because sometimes inequality (2.8) holds, but for the same real numbers $a$ and $b$, the inequality

$$
a \leq \phi(b)
$$

does not hold. To see this, let $\phi: \mathbb{R}^{+} \rightarrow \mathbb{R}^{+}$be a function defined by

$$
\phi(t)=\frac{\alpha t}{1+t}, \quad 0 \leq \alpha<1
$$

Obviously the function $\phi$ is continuous, nondecreasing and satisfies $\phi(t)=$ $\alpha t /(1+t)<t$ for $t>0$. Again since

$$
\sum_{n=1}^{\infty} \phi^{n}(t)=\sum_{n=1}^{\infty} \frac{\alpha^{n} t}{1+t+\cdots+\alpha^{n-1} t}<\sum_{n=1}^{\infty} \alpha^{n}<\infty,
$$

we have that $\phi \in \Phi$. 
Now for $a=1 / 2$ and $b=2 / 3$, we have, by (2.9),

$$
\frac{1}{2} \leq \phi\left(\frac{2}{3}\right)=\frac{(2 / 3) \alpha}{1+2 / 3}=\frac{2}{5} \alpha
$$

which is not true since $0 \leq \alpha<1$. But for the same values of $a$ and $b$, we have a positive real number $r=2$ such that

$$
\left(\frac{1}{2}\right)^{2}=\frac{1}{4} \leq \frac{4 \alpha}{13}=\phi\left(\left(\frac{2}{3}\right)^{2}\right)
$$

for $13 / 16 \leq \alpha<1$. Hence inequality (2.8) holds. Thus inequality (2.9) does not imply inequality (2.8). Actually, inequalities (2.8) and (2.9) are independent. To show that inequality (2.8) does not imply inequality (2.9), let $a=1 / 4, b=4 / 9$, and $r=1 / 2$. Clearly, inequality (2.8) does not hold, but for the same values of $a, b$, and $r$, one has

$$
\frac{1}{4} \leq 4 \frac{\alpha}{13}=\frac{\alpha(4 / 9)}{1+4 / 9}=\phi\left(\frac{4}{9}\right)
$$

for $\alpha \geq 13 / 16$, and so inequality (2.9) holds. Thus inequalities (2.8) and (2.9) are independent.

In the following sections, we will prove the main results of this paper.

3. Weak commuting mappings in $D$-metric spaces. Let $F: X \rightarrow 2^{X}$ and $g: X \rightarrow X$. Then the pair $\{F, g\}$ of maps is called limit coincident if $\lim _{n} F x_{n}=$ $\left\{\lim _{n} g x_{n}\right\}$ for some sequence $\left\{x_{n}\right\}$ in $X$, and coincident if there exists a point $u \in X$ such that $F u=\{g u\}$. Again two maps $F$ and $g$ are called limit commuting if $\lim _{n} F g x_{n}=\left\{\lim _{n} g F x_{n}\right\}$, where $\left\{x_{n}\right\}$ is a sequence in $X$, and commuting if $F g x=\{g F x\}$ for all $x \in X$. Two maps $F$ and $g$ are called limit coincidentally commuting if their limit coincidence implies the limit commutativity on $X$. Similarly, they are called coincidentally commuting if they are commuting at the coincidence points. Again two maps $F$ and $g$ are said to be limit pseudocommuting if $\lim _{n} F g x_{n} \cap \lim _{n} g F x_{n} \neq \phi$, that is, $\lim _{n} D\left(F g x_{n}, g F x_{n}, g F x_{n}\right)=0$, where $\left\{x_{n}\right\}$ is a sequence in $X$, and pseudocommuting if $F g x \cap g F x \neq \varnothing$ for each $x \in X$. Finally, the pair $\{F, g\}$ is called limit coincidentally pseudocommuting if its limit coincidence implies the limit pseudocommutativity on $X$, and coincidentally pseudocommuting if it is pseudocommuting at the coincidence points. It is known that a coincidentally commuting pair is limit coincidentally commuting and a coincidentally pseudocommuting pair is limit coincidentally pseudocommuting, but the converse implications need not hold. A pair of maps $\{F, g\}$ is weak commuting if it is either limit commuting, coincidentally commuting, limit pseudocommuting, or coincidentally pseudocommuting on $X$. Below, we will prove some common fixed-point theorems for each of these weak commuting mappings on $D$-metric spaces. 
3.1. Limit coincidentally commuting maps in $D$-metric spaces. Let $F: X \rightarrow$ $2^{X}$ and $g: X \rightarrow X$. By an $(F / g)$-orbit of the pair $\{F, g\}$ of maps at a point $x \in X$, we mean a set $O_{F}(g x)$ in $X$ defined by

$$
O_{F}(g x)=\left\{y_{n} \mid y_{0}=g x_{0}, y_{n}=g x_{n} \in F x_{n-1}, n \in \mathbb{N} \text {, where } x_{0}=x\right\}
$$

for some sequence $\left\{x_{n}\right\}$ in $X$. The orbit $O_{F}(g x)$ is well defined for each $x \in X$ if $F(X) \subseteq g(X)$. By $\overline{O_{F}(g x)}$ we denote the closure of the set $O_{F}(g x)$ in $X$.

A $D$-metric space $X$ is called $(F / g)$-orbitally bounded if $\delta\left(O_{F}(g x)\right)<\infty$ for each $x \in X$. Further $X$ is called $(F / g)$-orbitally complete if every $D$-Cauchy sequence $\left\{x_{n}\right\} \subset O_{F}(g x)$ converges to a point in $X$ for each $x \in X$. Finally, a mapping $T: X \rightarrow \mathrm{CB}(X)$ is called $(F / g)$-orbitally continuous if for any $\left\{x_{n}\right\} \subset$ $O_{F}(g x), x_{n} \rightarrow x^{*}$ implies that $T x_{n} \rightarrow T x^{*}$ for each $x \in X$.

THeOrem 3.1. Let $F: X \rightarrow \mathrm{CB}(X)$ and $g: X \rightarrow X$ be two mappings satisfying, for some positive real number $r$,

$$
\begin{aligned}
& \delta^{r}(F x, F y, F z) \\
& \leq \phi\left(\operatorname { m a x } \left\{\rho^{r}(g x, g y, g z), \delta^{r}(F x, F y, g z), \delta^{r}(g x, F x, g z),\right.\right. \\
&\left.\left.\delta^{r}(g y, F y, g z), \delta^{r}(g x, F y, g z), \delta^{r}(g y, F x, g z)\right\}\right)
\end{aligned}
$$

for all $x, y, z \in X$, where $\phi \in \Phi$. Suppose that

(a) $F(X) \subseteq g(X)$ and $g(X)$ is bounded,

(b) $\{F, g\}$ is limit coincidentally commuting,

(c) $F$ or $g$ is $(F / g)$-orbitally continuous.

Further if $X$ is $(F / g)$-orbitally complete $D$-metric space, then $F$ and $g$ have a unique common fixed point $u \in X$ such that $F u=\{u\}=g u$. Moreover, if $g$ is continuous at $u$, then $F$ is also continuous at $u$ in the Kasubai D-metric on $X$.

Proof. Let $x \in X$ be arbitrary and define a sequence $\left\{y_{n}\right\}$ in $X$ as follows. Take $x_{0}=x$ and $y_{0}=g x_{0}$. Choose a point $y_{1} \in F x_{0}=X_{1}$. Since $F(X) \subseteq g(X)$, there is a point $x_{1} \in X$ such that $y_{1}=g x_{1}$. Again choose a point $y_{2} \in F x_{1}=X_{2}$. By hypothesis (a), there is a point $x_{2} \in X$ such that $y_{2}=g x_{2}$. Proceeding in this way, by induction there is a sequence $\left\{x_{n}\right\}$ of points in $X$ such that

$$
y_{0}=g x_{0}, \quad y_{n+1}=g x_{n+1} \in X_{n+1}=F x_{n}, \quad n=0,1,2, \ldots
$$

From hypothesis (a), it follows that

$$
\delta\left(X_{m}, X_{n}, X_{p}\right) \leq \delta(g(X))=k<\infty
$$

for all $m, n, p \in \mathbb{N}$.

Now there are two cases.

CASE 1. Suppose that $y_{r}=y_{r+1}$ for some $r \in \mathbb{N}$. Then we have $g x_{r}=$ $g x_{r+1}=u$ for some $u \in X$. 
We will show that $F x_{r}=\{u\}$. Suppose not. Then by (3.2),

$$
\begin{aligned}
\delta^{r} & \left(F x_{r}, F x_{r}, u\right) \\
& =\delta^{r}\left(F x_{r}, F x_{r}, g x_{r+1}\right) \\
& \leq \delta^{r}\left(F x_{r}, F x_{r}, F x_{r}\right) \\
& \leq \phi\left(\max \left\{\rho^{r}\left(g x_{r}, g x_{r}, g x_{r}\right), \delta^{r}\left(g x_{r}, F x_{r}, g x_{r}\right), \delta^{r}\left(F x_{r}, F x_{r}, g x_{r}\right)\right\}\right) \\
& \leq \phi\left(\max \left\{0, \delta^{r}\left(g x_{r}, F x_{r}, g x_{r}\right), \delta^{r}\left(F x_{r}, F x_{r}, u\right)\right\}\right) \\
& =\phi\left(\max \left\{\delta^{r}\left(u, F x_{r}, u\right), \delta^{r}\left(F x_{r}, F x_{r}, u\right)\right\}\right) \\
& =\phi\left(\delta^{r}\left(u, F x_{r}, u\right)\right)
\end{aligned}
$$

because $\delta^{r}\left(F x_{r}, F x_{r}, u\right) \leq \phi\left(\delta^{r}\left(F x_{r}, F x_{r}, u\right)\right)$ is not possible in view of $\phi \in \Phi$. Again by (3.2),

$$
\begin{aligned}
\delta^{r}\left(F x_{r}, u, u\right) & =\delta^{r}\left(F x_{r}, g x_{r+1}, g x_{r+1}\right) \\
& \leq \delta^{r}\left(F x_{r}, F x_{r}, F x_{r}\right) \\
& \leq \phi\left(\max \left\{\delta^{r}\left(u, F x_{r}, u\right), \delta^{r}\left(F x_{r}, F x_{r}, u\right)\right\}\right) \\
& =\phi\left(\delta^{r}\left(F x_{r}, F x_{r}, u\right)\right) .
\end{aligned}
$$

Substituting (3.6) in (3.5), we obtain

$$
\delta^{r}\left(F x_{r}, F x_{r}, u\right) \leq \phi^{2}\left(\delta^{r}\left(F x_{r}, F x_{r}, u\right)\right)
$$

which is a contradiction since $\phi \in \Phi$. Hence $F x_{r}=u$. Since $F$ and $g$ are limit coincidentally commuting, one has $F g x_{r}=\left\{g F x_{r}\right\}$.

We will show that $u$ is a common fixed point of $F$ and $g$ such that $F u=$ $\{u\}=g u$.

Now,

$$
\begin{aligned}
\delta^{r}(F u, g u, u)= & \delta^{r}\left(F F x_{r}, F g x_{r}, F x_{r}\right) \\
\leq & \phi\left(\operatorname { m a x } \left\{\rho^{r}\left(g F x_{r}, g g x_{r}, g x_{r}\right), \delta^{r}\left(F F x_{r}, F g x_{r}, g x_{r}\right),\right.\right. \\
& \quad \delta^{r}\left(g F x_{r}, F F x_{r}, g x_{r}\right), \delta^{r}\left(g g x_{r}, F g x_{r}, g x_{r}\right), \\
& \left.\left.\quad \delta^{r}\left(g F x_{r}, F g x_{r}, g x_{r}\right), \delta^{r}\left(g g x_{r}, F F x_{r}, g x_{r}\right)\right\}\right) \\
= & \phi\left(\max \left\{\rho^{r}\left(g F x_{r}, g g x_{r}, g x_{r}\right), \delta^{r}\left(g g x_{r}, F F x_{r}, g x_{r}\right)\right\}\right) \\
= & \phi\left(\delta^{r}(F u, g u, u)\right),
\end{aligned}
$$

which is possible only when $F u=\{u\}=g u$ since $\phi \in \Phi$.

CASE 2. Assume that $y_{n} \neq y_{n+1}$ for each $n \in \mathbb{N}$. We will show that $\left\{y_{n}\right\}$ is a $D$-Cauchy sequence in $X$. Let $x=x_{0}, y=x_{1}$, and $z=x_{m-1}, m \geq 1$. Then by (3.2), 


$$
\begin{aligned}
& \rho^{r}\left(y_{1}, y_{2}, y_{m}\right) \\
& \leq \delta^{r}\left(F x_{0}, F x_{1}, F x_{m-1}\right) \\
& \leq \phi\left(\operatorname { m a x } \left\{\rho^{r}\left(g x_{0}, g x_{1}, g x_{m-1}\right), \delta^{r}\left(F x_{0}, F x_{1}, g x_{m-1}\right), \delta^{r}\left(g x_{0}, F x_{0}, g x_{m-1}\right),\right.\right. \\
& \left.\left.\quad \delta^{r}\left(g x_{1}, F x_{1}, g x_{m-1}\right), \delta^{r}\left(g x_{0}, F x_{1}, g x_{m-1}\right), \delta^{r}\left(g x_{1}, F x_{0}, g x_{m-1}\right)\right\}\right) \\
& \leq \phi\left(\operatorname { m a x } \left\{\delta^{r}\left(X_{0}, X_{1}, X_{m-1}\right), \delta^{r}\left(X_{1}, X_{2}, X_{m-1}\right), \delta^{r}\left(X_{0}, X_{1}, X_{m-1}\right),\right.\right. \\
& \left.\left.\quad \delta^{r}\left(X_{1}, X_{2}, X_{m-1}\right), \delta^{r}\left(X_{0}, X_{2}, X_{m-1}\right), \delta^{r}\left(X_{1}, X_{1}, X_{m-1}\right)\right\}\right) \\
& \leq \phi\left(\max _{0 \leq a \leq 1,1 \leq b \leq 2} \delta^{r}\left(X_{a}, X_{b}, X_{m-1}\right)\right) \\
& \leq \phi\left(k^{r}\right),
\end{aligned}
$$

that is,

$$
\rho\left(y_{1}, y_{2}, y_{m}\right) \leq\left[\phi\left(k^{r}\right)\right]^{1 / r} .
$$

Similarly, letting $x=x_{1}, y=x_{2}$, and $z=z_{m-1}, m \geq 2$ in (3.2), we obtain

$$
\begin{aligned}
\rho^{r}( & \left.y_{2}, y_{3}, y_{m}\right) \\
\leq & \delta^{r}\left(F x_{1}, F x_{2}, F x_{m-1}\right) \\
\leq & \phi\left(\operatorname { m a x } \left\{\rho^{r}\left(g x_{1}, g x_{2}, g x_{m-1}\right), \delta^{r}\left(F x_{1}, F x_{2}, g x_{m-1}\right),\right.\right. \\
& \delta^{r}\left(g x_{1}, F x_{1}, g x_{m-1}\right), \delta^{r}\left(g x_{2}, F x_{2}, g x_{m-1}\right), \\
& \left.\left.\delta^{r}\left(g x_{1}, F x_{2}, g x_{m-1}\right), \delta^{r}\left(g x_{2}, F x_{1}, g x_{m-1}\right)\right\}\right) \\
\leq & \phi\left(\operatorname { m a x } \left\{\delta^{r}\left(F x_{0}, F x_{1}, F x_{m-2}\right), \delta^{r}\left(F x_{1}, F x_{2}, F x_{m-2}\right),\right.\right. \\
& \delta^{r}\left(F x_{0}, F x_{1}, F x_{m-2}\right), \delta^{r}\left(F x_{1}, F x_{2}, F x_{m-2}\right), \\
\leq & \left.\left.\delta^{r}\left(F x_{0}, F x_{2}, F x_{m-2}\right), \delta^{r}\left(F x_{1}, F x_{1}, F x_{m-2}\right)\right\}\right) \\
\leq & \phi\left(\phi\left(k_{0 \leq a \leq 2,1 \leq b \leq 3}\right)\right) \\
= & \phi^{2}\left(k^{r}\right),
\end{aligned}
$$

that is,

$$
\rho\left(y_{2}, y_{3}, y_{m}\right) \leq\left[\phi^{2}\left(k^{r}\right)\right]^{1 / r} .
$$

In general, by induction,

$$
\rho\left(y_{n}, y_{n+1}, y_{m}\right) \leq\left[\phi^{n}\left(k^{r}\right)\right]^{1 / r}
$$

for all $m>n \in \mathbb{N}$.

Hence, the application of Lemma 2.2 yields that $\left\{y_{n}\right\}$ is a $D$-Cauchy sequence in $X$. The $D$-metric space $X$ being complete, there is a point $u \in X$ such that $\lim _{n} y_{n}=u$. The definition of $\left\{y_{n}\right\}$ implies that $\lim _{n} g x_{n}=u$. We will show that $\lim _{n} F x_{n}=\{u\}$. 
Now,

$$
\begin{aligned}
\lim _{n} & \delta^{r}\left(F x_{n}, F x_{n}, u\right) \\
& =\lim _{n} \delta^{r}\left(F x_{n}, F x_{n}, y_{n+1}\right) \\
& \leq \lim _{n} \delta^{r}\left(F x_{n}, F x_{n}, F x_{n}\right) \\
& \leq \lim _{n} \phi\left(\max \left\{\rho^{r}\left(g x_{n}, g x_{n}, g x_{n}\right), \delta^{r}\left(F x_{n}, F x_{n}, g x_{n}\right), \delta^{r}\left(g x_{n}, F x_{n}, g x_{n}\right)\right\}\right) \\
& =\lim _{n} \phi\left(\max \left\{\delta^{r}\left(F x_{n}, F x_{n}, u\right), 0\right\}\right) \\
& =\phi\left(\lim _{n} \delta^{r}\left(F x_{n}, F x_{n}, u\right)\right),
\end{aligned}
$$

which implies that $\lim _{n} F x_{n}=u$. Thus we have

$$
\lim _{n} F x_{n}=\{u\}=\lim _{n} g x_{n} .
$$

Since $F$ and $g$ are limit coincidentally commuting, one has

$$
\lim _{n} F g x_{n}=\left\{\lim _{n} g F x_{n}\right\} .
$$

Suppose that $g$ is $(F / g)$-orbitally continuous on $X$. Then we have

$$
\lim _{n} F \mathfrak{g} x_{n}=\lim _{n} g F x_{n}=\lim _{n} g g x_{n}=g u .
$$

First, we will show that $u$ is a common fixed point of $F$ and $g$. Suppose not. Then we have

$$
\begin{aligned}
\delta^{r}(u, u, g u) & =\lim _{n} \delta^{r}\left(F x_{n}, F x_{n}, g F x_{n}\right) \\
& =\lim _{n} \delta^{r}\left(F x_{n}, F x_{n}, F g x_{n}\right) \\
\leq & \lim _{n} \phi\left(\operatorname { m a x } \left\{\rho^{r}\left(g x_{n}, g x_{n}, g g x_{n}\right),\right.\right. \\
& \left.\left.\quad \delta^{r}\left(F x_{n}, F x_{n}, g g x_{n}\right), \delta^{r}\left(g x_{n}, F x_{n}, g g x_{n}\right)\right\}\right) \\
= & \phi\left(\max \left\{\lim _{n} \delta^{r}\left(g x_{n}, g x_{n}, g g x_{n}\right), \lim _{n} \delta^{r}\left(F x_{n}, F x_{n}, g g x_{n}\right)\right\}\right) \\
& =\phi\left(\delta^{r}(u, u, g u)\right),
\end{aligned}
$$

which is a contradiction and hence $g u=u$.

Again

$$
\begin{aligned}
& \delta^{r}(F u, g u, u) \\
& =\lim _{n} \delta^{r}\left(F u, F x_{n}, F g x_{n}\right) \\
& \leq \lim _{n} \phi\left(\operatorname { m a x } \left\{\rho^{r}\left(g u, g x_{n}, g g x_{n}\right), \delta^{r}\left(F u, F x_{n}, g g x_{n}\right), \delta^{r}\left(g u, F u, g g x_{n}\right),\right.\right. \\
& \left.\left.\quad \delta^{r}\left(g x_{n}, F x_{n}, g g x_{n}\right), \delta^{r}\left(g u, F x_{n}, g g x_{n}\right), \delta^{r}\left(g x_{n}, F u, g g x_{n}\right)\right\}\right)
\end{aligned}
$$




$$
\begin{gathered}
=\phi\left(\operatorname { m a x } \left\{\rho^{r}(g u, u, g u), \delta^{r}(F u, u, g u), \delta^{r}(g u, F u, g u),\right.\right. \\
\left.\left.\delta^{r}(u, u, g u), \delta^{r}(g u, u, g u), \delta^{r}(u, F u, g u)\right\}\right) \\
=\phi\left(\delta^{r}(F u, g u, u)\right),
\end{gathered}
$$

which is possible only when $F u=\{u\}=g u$ since $\phi \in \Phi$. Thus $u$ is a common fixed point of $F$ and $g$.

Next, suppose that $F$ is $(F / g)$-orbitally continuous on $X$. Then we have

$$
\lim _{n} F g x_{n}=\lim _{n} g F x_{n}=\lim _{n} F F x_{n}=F u=\{z\}
$$

We will show that $z$ is a common fixed point of $F$ and $g$. Since $F(X) \subseteq g(X)$, there is a point $v \in X$ such that $F u=g v=z$. We will show that $F v=g v=\{z\}$. By (3.2),

$$
\begin{aligned}
\delta^{r}(F v, g v, F v) \\
\quad=\lim _{n} \delta^{r}\left(F v, F v, F F x_{n}\right) \\
\quad \leq \lim _{n} \phi\left(\max \left\{\rho^{r}\left(g v, g v, g F x_{n}\right), \delta^{r}\left(F v, g v, g F x_{n}\right), \delta^{r}\left(g v, F v, g F x_{n}\right)\right\}\right) \\
\quad=\phi\left(\max \left\{\delta^{r}(g v, g v, g v), \delta^{r}(F v, g v, z)\right\}\right),
\end{aligned}
$$

that is,

$$
\delta^{r}(F v, g v, z) \leq \phi\left(\delta^{r}(F v, g v, z)\right)
$$

which implies that $F v=g v=\{z\}$ since $\phi \in \Phi$.

Since $F$ and $g$ are limit coincidentally commuting, they are coincidentally commuting on $X$. Therefore, we have $F g v=g F v$. Now, proceeding with the arguments as in Case 1, it is proved that $z$ is a common fixed point of $F$ and $g$.

To prove the uniqueness, let $z^{*}(\neq z)$ be another common fixed point of $F$ and $g$. Then by (3.2),

$$
\begin{aligned}
\rho^{r}\left(z, z, z^{*}\right) & =\delta^{r}\left(F z, F z, F z^{*}\right) \\
& \leq \phi\left(\operatorname { m a x } \left\{\rho^{r}\left(g z, g z, g z^{*}\right), \delta^{r}\left(F z, F z, g z^{*}\right)\right.\right. \\
& \left.\left.\delta^{r}\left(g z, F z, g z^{*}\right), \delta^{r}\left(g z, F z, g z^{*}\right)\right\}\right) \\
& =\phi\left(\rho^{r}\left(z, z, z^{*}\right)\right)
\end{aligned}
$$

which is a contradiction. Hence $z=z^{*}$. Then $F$ and $g$ have a unique common fixed point $z \in X$ with $F z=\{z\}=g z$.

Finally, suppose that $g$ is continuous at the common fixed point $z$ of $F$ and $g$. Then we will prove that $F$ is also continuous at $z$. Let $\left\{z_{n}\right\}$ be any sequence 
in $X$ converging to the common fixed point $z$. Since $g$ is continuous on $X$, we have

$$
\lim _{m, n} \rho\left(z_{m}, z_{n}, z\right)=0 \Longrightarrow \lim _{m, n} \rho\left(g z_{m}, g z_{n}, g z\right)=0 .
$$

From (1.2), it follows that

$$
\kappa\left(F z_{m}, F z_{n}, F z\right) \leq \delta\left(F z_{m}, F z_{n}, F z\right) .
$$

Now,

$$
\begin{aligned}
\delta^{r}\left(F z_{m}, F z_{n}, F z\right) \\
\leq \phi\left(\operatorname { m a x } \left\{\rho^{r}\left(g z_{m}, g z_{n}, g z\right), \delta^{r}\left(F z_{m}, F z_{n}, g z\right), \delta^{r}\left(g z_{m}, F z_{m}, g z\right)\right.\right. \\
\left.\left.\delta^{r}\left(g z_{n}, F z_{n}, g z\right), \delta^{r}\left(g z_{m}, F z_{n}, g z\right), \delta^{r}\left(g z_{n}, F z_{m}, g z\right)\right\}\right) .
\end{aligned}
$$

Therefore,

$$
\begin{aligned}
\lim _{m, n} \delta^{r}\left(F z_{m}, F z_{n}, F z\right) & \\
\leq & \lim _{m, n} \phi\left(\operatorname { m a x } \left\{\rho^{r}\left(g z_{m}, g z_{n}, g z\right), \delta^{r}\left(F z_{m}, F z_{n}, F z\right), \delta^{r}\left(g z_{m}, F z_{m}, z\right),\right.\right. \\
& \left.\left.\delta^{r}\left(g z_{n}, F z_{n}, z\right), \delta^{r}\left(g z_{m}, F z_{n}, z\right), \delta^{r}\left(g z_{n}, F z_{m}, z\right)\right\}\right) \\
= & \phi\left(\max \left\{0, \lim _{m, n} \delta^{r}\left(F z_{m}, F z_{n}, F z\right), \lim _{m} \delta^{r}\left(z, F z_{m}, z\right), \lim _{n} \delta^{r}\left(z, F z_{n}, z\right)\right\}\right) \\
= & \phi\left(\max \left\{\lim _{m} \delta^{r}\left(z, F z_{m}, z\right), \lim _{n} \delta^{r}\left(z, F z_{n}, z\right)\right\}\right) .
\end{aligned}
$$

But

$$
\begin{aligned}
\lim _{m} & \delta^{r}\left(z, F z_{m}, z\right) \\
\quad & =\lim _{m} \delta^{r}\left(F z, F z, F z_{m}\right) \\
& \leq \lim _{m} \phi\left(\max \left\{\rho^{r}\left(g z, g z, g z_{m}\right), \delta^{r}\left(F z, F z, g z_{m}\right), \delta^{r}\left(g z, F z, g z_{m}\right)\right\}\right) \\
& =\phi(\max \{0,0,0\}) \\
& =0 .
\end{aligned}
$$

Similarly, $\lim _{n} \delta^{r}\left(z, F z_{n}, z\right)=0$. Substituting these estimates in (3.27) yields that

$$
\lim _{m, n} \delta^{r}\left(F z_{m}, F z_{n}, F z\right)=0
$$

or

$$
\lim _{m, n} \delta\left(F z_{m}, F z_{n}, F z\right)=0
$$

Now from (3.25), it follows that

$$
\lim _{m, n} \kappa\left(F z_{m}, F z_{n}, F z\right)=0,
$$


and so $F$ is continuous at the common fixed point $z$ of $F$ and $g$. This completes the proof.

Letting $g=I$, the identity map on $X$ and $r=1$, in Theorem 3.1, we obtain the following corollary.

COROLlARY 3.2. Let $F: X \rightarrow \mathrm{CB}(X)$ be a multivalued mapping satisfying

$$
\begin{array}{r}
\delta(F x, F y, F z) \leq \phi(\rho(x, y, z), \delta(F x, F y, z), \delta(x, F x, z), \\
\delta(y, F y, z), \delta(x, F y, z), \delta(y, F x, z))
\end{array}
$$

for all $x, y, z \in X$, where $\phi \in \Phi$. Further if $X$ is $F$-orbitally bounded and $F$ orbitally complete $D$-metric space, then $F$ has a unique fixed point $u \in X$ such that $F u=\{u\}$ and $F$ is continuous at $u$.

COROLlaRY 3.3. Let $F: X \rightarrow \mathrm{CB}(X)$ be a multivalued mapping satisfying

$$
\begin{array}{r}
\delta(F x, F y, F z) \leq \lambda \max \{\rho(x, y, z), \delta(F x, F y, z), \delta(x, F x, z), \\
\delta(y, F y, z), \delta(x, F y, z), \delta(y, F x, z)\}
\end{array}
$$

for all $x, y, z \in X$, where $0 \leq \lambda<1$. Further if $X$ is $F$-orbitally bounded and $F$-orbitally complete $D$-metric space, then $F$ has a unique fixed point $u \in X$ such that $F u=\{u\}$ and $F$ is continuous at $u$.

Corollary 3.3 includes the following fixed point of Dhage [3] as a special case.

COROLlary 3.4 (see [3]). Let $X$ be a bounded and complete D-metric space and let $F: X \rightarrow \mathrm{CB}(X)$ be a multivalued mapping satisfying

$$
\delta(F x, F y, F z) \leq \lambda \rho(x, y, z)
$$

for all $x, y, z \in X$, where $0 \leq \lambda<1$. Then $F$ has a unique fixed point $u \in X$ such that $F u=\{u\}$ and $F$ is continuous at $u$.

COROLlary 3.5. Let $f, g: X \rightarrow X$ be two mappings satisfying

$$
\begin{array}{r}
\rho^{r}(f x, f y, f z) \\
\leq \phi\left(\operatorname { m a x } \left\{\rho^{r}(g x, g y, g z), \rho^{r}(f x, f y, g z), \rho^{r}(g x, f x, g z),\right.\right. \\
\left.\left.\rho^{r}(g y, f y, g z), \rho^{r}(g x, f y, g z), \rho^{r}(g y, f x, g z)\right\}\right)
\end{array}
$$

for all $x, y, z \in X$, where $\phi \in \Phi$. Suppose that

(a) $f(X) \subseteq g(X)$,

(b) $\{f, g\}$ is limit coincidentally commuting,

(c) $f$ or $g$ is continuous.

Further if $X$ is $(f / g)$-orbitally bounded and $(f / g)$-orbitally complete D-metric space, then $f$ and $g$ have a unique common fixed point $u \in X$. Moreover, if $g$ is continuous at $u$, then $f$ is also continuous at $u$. 
REMARK 3.6. Note that Corollary 3.5 includes the class of pairs of fixedpoint mappings of Dhage [7] characterized by the inequality

$$
\begin{aligned}
& \rho^{r}(f x, f y, f z) \\
& \leq \phi\left(\operatorname { m a x } \left\{\rho^{r}(g x, g y, g z), \rho^{r}(g x, f x, g z),\right.\right. \\
& \left.\left.\rho^{r}(g y, f y, g z), \rho^{r}(g x, f y, g z), \rho^{r}(g y, f x, g z)\right\}\right)
\end{aligned}
$$

for all $x, y, z \in X$ and $\phi \in \Phi$.

COROLlary 3.7. Let $f, g: X \rightarrow X$ be two mappings satisfying for some positive real numbers $p, q$, and $r$,

$$
\begin{array}{r}
\rho^{r}\left(f^{p} x, f^{p} y, f^{p} z\right) \\
\leq \phi\left(\operatorname { m a x } \left\{\rho^{r}\left(g^{q} x, g^{q} y, g^{q} z\right), \rho^{r}\left(f^{p} x, f^{p} y, g^{q} z\right),\right.\right. \\
\rho^{r}\left(g^{q} x, f^{p} x, g^{q} z\right), \rho^{r}\left(g^{q} y, f^{p} y, g^{q} z\right), \\
\left.\left.\rho^{r}\left(g^{q} x, f^{p} y, g^{q} z\right), \rho^{r}\left(g^{q} y, f^{p} x, g^{q} z\right)\right\}\right)
\end{array}
$$

for all $x, y, z \in X$, where $\phi \in \Phi$. Suppose that

(a) $f^{p}(X) \subseteq g^{q}(X)$,

(b) $\{f, g\}$ is commuting,

(c) $f$ or $g$ is continuous.

Further if $X$ is an $\left(f^{p} / g^{q}\right)$-orbitally bounded and $\left(f^{p} / g^{q}\right)$-orbitally complete $D$-metric space, then $f$ and $g$ have a unique common fixed point $u \in X$. Moreover, if $g$ is continuous at $u$, then $f^{p}$ is also continuous at $u$.

Proof. Let $S=f^{p}$ and $T=g^{q}$. Then by Corollary 3.5, $S$ and $T$ have a unique common fixed point $u \in X$, that is, $S u=f^{p} u=u=g^{q} u=T u$. Now by commutativity of $f$ and $g$, we obtain

$$
f u=f\left(f^{p} u\right)=f^{p}(f u), \quad f u=f\left(g^{q} u\right)=g^{q}(f u) .
$$

This shows that $f u$ is again a common fixed point of $f^{p}$ and $g^{q}$. By the uniqueness of $u$, we have $f u=u$. Similarly it is proved that $g u=u$. Thus $f$ and $g$ have a unique common fixed point $u \in X$. Further if $g$ is continuous on $X, g^{q}$ is continuous on $X$ and by application of Corollary 3.5 yields that $f^{p}$ is continuous at $u$. This completes the proof.

Corollary 3.7 includes the class of pairs of fixed-point mappings of Dhage [7] characterized by the inequality

$$
\begin{aligned}
& \rho^{r}\left(f^{p} x, f^{p} y, f^{p} z\right) \\
& \leq \phi\left(\operatorname { m a x } \left\{\rho^{r}\left(g^{q} x, g^{q} y, g^{q} y\right), \rho^{r}\left(g^{q} x, f^{p} x, g^{q} z\right),\right.\right. \\
& \left.\left.\quad \rho^{r}\left(g^{q} y, f^{p} y, g^{q} z\right), \rho^{r}\left(g^{q} x, f^{p} y, g^{q} z\right), \rho^{r}\left(g^{q} y, f^{p} x, g^{q} z\right)\right\}\right)
\end{aligned}
$$

for all $x, y, z \in X$ and $\phi \in \Phi$. 
COROLLARY 3.8. Let $f$ be a self-map of a D-metric space $X$ satisfying

$$
\begin{array}{r}
\rho(f x, f y, f z) \leq \lambda \max \{\rho(x, y, z), \rho(f x, f y, z), \rho(x, f x, z), \\
\rho(y, f y, z), \rho(x, f y, z), \rho(y, f x, z)\}
\end{array}
$$

for all $x, y, z \in X$, where $0 \leq \lambda<1$. Further if $X$ is $f$-orbitally bounded and $f$-orbitally complete, then $f$ has a unique fixed point $u \in X$ and $f$ is continuous at $u$.

COROLlary 3.9. Let $f$ be a self-map of a $D$-metric space $X$ satisfying, for some positive real number $p$,

$$
\begin{array}{r}
\rho\left(f^{p} x, f^{p} y, f^{p} z\right) \\
\leq \lambda \max \left\{\rho(x, y, z), \rho\left(f^{p} x, f^{p} y, z\right), \rho\left(x, f^{p} x, z\right),\right. \\
\left.\rho\left(y, f^{p} y, z\right), \rho\left(x, f^{p} y, z\right), \rho\left(y, f^{p} x, z\right)\right\}
\end{array}
$$

for all $x, y, z \in X$, where $0 \leq \lambda<1$. Further if $X$ is $f$-orbitally bounded and $f$-orbitally complete, then $f$ has a unique fixed point $u \in X, f^{p}$ is continuous, and $f$ is $f$-orbitally continuous at $u$.

Note that Corollaries 3.8 and 3.9 include the fixed-point theorems of Rhoades [12] and Dhage [9] for the mappings characterized by the inequalities

$$
\begin{aligned}
\rho(f x, f y, f z) \leq \lambda \max \{ & \rho(x, y, z), \rho(x, f x, z), \\
& \rho(y, f y, z), \rho(x, f y, z), \rho(y, f x, z)\}, \\
\rho\left(f^{p} x, f^{p} y, f^{p} z\right) \leq \lambda \max \{ & \rho(x, y, z), \rho\left(x, f^{p} x, z\right), \\
& \left.\rho\left(y, f^{p} y, z\right), \rho\left(x, f^{p} y, z\right), \rho\left(y, f^{p} x, z\right)\right\},
\end{aligned}
$$

for all $x, y, z \in X$ and $0 \leq \lambda<1$.

3.2. Coincidentally commuting mappings. The coincidentally commuting mappings require some stronger condition than limit coincidentally commuting mappings and a good number of mathematicians have studied them on metric and $D$-metric spaces for the existence of their common fixed point. See $[5,11]$ and the references therein. The novelty of the fixed-point theorems for these coincidentally commuting mappings lies in the fact that here we do not require any of the maps under consideration to be continuous. Below, we prove a result in this direction and derive some interesting corollaries.

THeOrem 3.10. Let $X$ be a $D$-metric space and let $F: X \rightarrow \mathrm{CB}(X)$ and $g: X \rightarrow$ $X$ be two mappings satisfying (3.2). Further suppose that

(a) $F(X) \subseteq g(X)$,

(b) $g(X)$ is bounded and complete,

(c) $\{F, g\}$ is coincidentally commuting. 
Then $F$ and $g$ have a unique common fixed point $u \in X$ such that $F u=\{u\}=$ $g u$. Moreover, if $g$ is continuous at $u$, then $F$ is also continuous at $u$ in the Kasubai D-metric on $X$.

Proof. Let $x \in X$ be arbitrary and define a sequence $\left\{y_{n}\right\} \subset X$ by (3.3). Clearly the sequence $\left\{y_{n}\right\}$ is well defined since $F(X) \subseteq g(X)$. Further we note that $\left\{y_{n}\right\} \subseteq g(X)$. We prove the conclusion of the theorem in two cases.

CASE 1. Suppose that $y_{r}=y_{r+1}$ for some $r \in \mathbb{N}$. Then proceeding with the arguments similar to Case 1 of the proof of Theorem 3.1, it is proved that $y_{r}=u$ is a common fixed point of $F$ and $g$ such that $F u=\{u\}=g u$.

CASE 2. Assume that $y_{n} \neq y_{n+1}$ for each $n \in \mathbb{N}$. Then following Case 2 of the proof of Theorem 3.1, it is shown that $\left\{y_{n}\right\}$ is a $D$-Cauchy sequence. Since $g(X)$ is complete, there is a point $z \in g(X) \operatorname{such}$ that $\lim _{n} y_{n}=z=\lim _{n} g x_{n}$. We will show that $\lim _{n} F x_{n}=\{z\}$.

Now,

$$
\begin{aligned}
\lim _{n} & \delta^{r}\left(F x_{n}, F x_{n}, z\right) \\
& =\lim _{n} \delta^{r}\left(F x_{n}, F x_{n}, y_{n+1}\right) \\
& \leq \lim _{n} \delta^{r}\left(F x_{n}, F x_{n}, F x_{n}\right) \\
& \leq \lim _{n} \phi\left(\max \left\{\rho^{r}\left(g x_{n}, g x_{n}, g x_{n}\right), \delta^{r}\left(F x_{n}, F x_{n}, g x_{n}\right), \delta^{r}\left(g x_{n}, F x_{n}, g x_{n}\right)\right\}\right) \\
& =\phi\left(\max \left\{0, \lim _{n} \delta^{r}\left(F x_{n}, F x_{n}, z\right)\right\}\right) \\
& =\phi\left(\lim _{n} \delta^{r}\left(F x_{n}, F x_{n}, z\right)\right),
\end{aligned}
$$

which gives that $\lim _{n} F x_{n}=\{z\}$.

Since $z \in g(X)$, there is a point $u \in X$ such that $g u=u$. We will show that $F u=\{z\}=g u$. Now,

$$
\begin{aligned}
\delta^{r}(F u, z, z) \\
\quad=\lim _{n} \delta^{r}\left(F u, F x_{n}, F x_{n}\right) \\
\quad=\lim _{n} \delta^{r}\left(F x_{n}, F x_{n}, F u\right) \\
\quad \leq \lim _{n} \phi\left(\max \left\{\rho^{r}\left(g u, g x_{n}, g x_{n}\right), \delta^{r}\left(F x_{n}, F x_{n}, g u\right), \delta^{r}\left(g x_{n}, F x_{n}, g u\right)\right\}\right) \\
\quad=\phi(\max \{0,0,0\}) \\
\quad=\phi(0) \\
\quad=0
\end{aligned}
$$

and so $F u=g u=\{z\}$. Thus $u$ is a coincidence point of $F$ and $g$. The rest of the proof is similar to Case 2 of the proof of Theorem 3.1. We omitted the details. 
As a consequence of Theorem 3.10, we obtain the following corollaries.

Corollary 3.11. Let $f, g: X \rightarrow X$ be two mappings satisfying (3.35). Suppose that

(a) $f(X) \subseteq g(X)$,

(b) $g(X)$ is bounded and complete,

(c) $\{f, g\}$ is coincidentally commuting.

Then $f$ and $g$ have a unique common fixed point $u$ and if $g$ is continuous at $u$, then $f$ is also continuous at $u$.

Corollary 3.12. Let $X$ be a $D$-metric space and let $f, g: X \rightarrow X$ be two mappings satisfying

$$
\begin{array}{r}
\rho(f x, f y, f z) \\
\leq \lambda \max \{\rho(g x, g y, g z), \rho(f x, f y, g z), \rho(g x, f x, g z), \\
\rho(g y, f y, g z), \rho(g x, f y, g z), \rho(g y, f x, g z)\}
\end{array}
$$

for all $x, y, z \in X$, where $0 \leq \lambda<1$. Further suppose that hypotheses (a), (b), and (c) of Corollary 3.11 hold. Then $f$ and $g$ have a unique common fixed point $u \in X$ and if $g$ is continuous at $u$, then $f$ is also continuous at $u$.

Corollary 3.12 includes a common fixed-point theorem of Dhage [5] for the mappings $f$ and $g$ on a $D$-metric space characterized by the inequality

$$
\begin{aligned}
& \rho(f x, f y, g z) \\
& \leq \lambda \max \{\rho(g x, g y, g z), \rho(g x, f x, g z), \\
& \rho(g y, f y, g z), \rho(g x, f y, g z), \rho(g y, f x, g z)\}
\end{aligned}
$$

for all $x, y, z \in X$ and $0 \leq \lambda<1$.

Corollary 3.13. Let $X$ be a $D$-metric space and let $f, g: X \rightarrow X$ be two mappings satisfying (3.37). Further suppose that

(a) $f^{p}(X) \subseteq g^{q}(X)$,

(b) $g^{p}(X)$ is bounded and complete,

(c) $\{f, g\}$ is commuting.

Then $f$ and $g$ have a unique common fixed point $u$ and if $g^{q}$ is continuous at $u$, then $f^{p}$ is also continuous at $u$.

Notice that Corollary 3.13 includes a class of common fixed-point mappings $f$ and $g$ on a $D$-metric space $X$ characterized by the inequality

$$
\begin{aligned}
& \rho\left(f^{p} x, f^{p} y, f^{p} z\right) \\
& \leq \lambda \max \left\{\rho\left(g^{q} x, g^{q} y, g^{q} z\right), \rho\left(g^{q} x, f^{p} x, g^{q} z\right),\right. \\
& \left.\rho\left(g^{q} y, f^{p} y, g^{q} z\right), \rho\left(g^{q} x, f^{p} y, g^{q} z\right), \rho\left(g^{q} y, f^{p} x, g^{q} z\right)\right\}
\end{aligned}
$$

for all $x, y, z \in X$ and $0 \leq \lambda<1$. See [5]. 
4. Weak commuting mappings in compact $D$-metric spaces. In this section, we prove some common fixed-point theorems for the pairs of singlevalued and multivalued coincidentally commuting mappings on a $D$-metric space satisfying a contraction condition more general than (4.3). But in this case the $D$-metric space under consideration is required to satisfy a stronger condition of compactness and the mappings under consideration are required to satisfy the continuity condition on the $D$-metric spaces. Our results of this section generalize some earlier known fixed-point theorems such as those of Dhage [9] and Rhoades [12] for single maps as well as for a pair of maps on $D$-metric spaces.

THEOREM 4.1. Let $X$ be a compact $D$-metric space and let $F: X \rightarrow \mathrm{CB}(X)$ and $g: X \rightarrow X$ be two continuous mappings satisfying, for some positive real number $r$,

$$
\begin{aligned}
\delta^{r}(F x, F y, F z) \\
<\max \left\{\rho^{r}(g x, g y, g z), \delta^{r}(F x, F y, g z), \delta^{r}(g x, F x, g z),\right. \\
\left.\delta^{r}(g y, F y, g z), \delta^{r}(g x, F y, g z), \delta^{r}(g y, F x, g z)\right\}
\end{aligned}
$$

for all $x, y, z \in X$ for which the right-hand side is not zero. Further suppose that

(a) $F(X) \subseteq g(X)$,

(b) $\{F, g\}$ is limit coincidentally commuting.

Then $F$ and $g$ have a unique common fixed point $u \in X$ such that $F u=\{u\}=$ gu.

Proof. From inequality (4.3), it follows that if $F$ and $g$ have a common fixed point $u \in X$, then it is unique and $F u=\{u\}=g u$. Since $X$ is compact and $\delta$ is continuous, both sides of inequality (4.1) are bounded on $X$. Now, there are two cases.

CASE 1. Suppose that the right-hand side of (4.1) is zero for some $x, y, z \in$ $X$. Then, we have

$$
F x=g x=g z, \quad F y=g y=g z .
$$

Now, proceeding with the arguments similar to Case 1 of the proof of Theorem 3.1, it is proved that $u=F x=g x$ is a common fixed point of $F$ and $g$ and so it is unique.

CASE 2. Suppose that the right-hand side of inequality (4.1) is not zero for all $x, y, z \in X$. Define a mapping $T: X \times X \times X \rightarrow(0, \infty)$ by

$$
T(x, y, z)=\frac{\delta^{r}(F x, F y, F z)}{M(x, y, z)}
$$

where

$$
\begin{aligned}
M(x, y, z)=\max \{ & \rho^{r}(g x, g y, g z), \delta^{r}(F x, F y, g z), \delta^{r}(g x, F x, g z), \\
& \left.\delta^{r}(g y, F y, g z), \delta^{r}(g x, F y, g z), \delta^{r}(g y, F x, g z)\right\} .
\end{aligned}
$$


Clearly, the function $T$ is well defined since $M(x, y, z) \neq 0$ for all $x, y, z \in X$. Since $F$ and $g$ are continuous, from the compactness of $X$ it follows that the function $T$ attains its maximum on $X^{3}$ at some point $u, v, w \in X$. Call the value $c$. It is clear from (4.1) that $0<c<1$. By the definition of $c$, we have $T(x, y, z) \leq c$ for all $x, y, z \in X$. This further, in view of (4.3), implies that

$$
\begin{aligned}
& \delta^{r}(F x, F y, F z) \\
& \quad \leq c M(x, y, z) \\
& =c \max \left\{\rho^{r}(g x, g y, g z), \delta^{r}(F x, F y, F z), \delta^{r}(g x, F x, g x),\right. \\
& \left.\quad \delta^{r}(g y, F y, g z), \delta^{r}(g x, F y, g z), \delta^{r}(g y, F x, g z)\right\}
\end{aligned}
$$

for all $x, y, z \in X$.

As $X$ is compact, it is complete and $g(X)$ is bounded in view of the continuity of $g$ on $X$. Now, the desired conclusion follows by an application of Theorem 3.1. This completes the proof.

Now we derive some interesting corollaries.

COROLlarY 4.2. Let $X$ be a compact $D$-metric space and let $F: X \rightarrow \mathrm{CB}(X)$ be a continuous mapping satisfying

$$
\begin{array}{r}
\delta(F x, F y, F z)<\max \{\rho(x, y, z), \delta(F x, F y, z), \delta(x, F x, z), \\
\delta(y, F y, z), \delta(x, F y, z), \delta(y, F x, z)\}
\end{array}
$$

for all $x, y, z \in X$ for which the right-hand side is not zero. Then $F$ has a unique fixed point $u \in X$ such that $F u=\{u\}$.

Proof. The proof follows by letting $g=I$ in Theorem 4.1, where $I$ is the identity map on $X$.

COROLlary 4.3 (see [3]). Let $X$ be a compact $D$-metric space and let $F: X \rightarrow$ $\mathrm{CB}(X)$ be a continuous mapping satisfying

$$
\delta(F x, F y, F z)<\rho(x, y, z)
$$

for all $x, y, z \in X$ for which $\rho(x, y, z) \neq 0$. Then $F$ has a unique fixed point $u \in X$ such that $F u=\{u\}$.

COROLlary 4.4. Let $X$ be a compact $D$-metric space and let $f, g: X \rightarrow X$ be two continuous mappings satisfying

$$
\begin{array}{r}
\rho(f x, f y, f z)<\max \{\rho(g x, g y, g z), \rho(f x, f y, g z), \rho(g x, f x, g z), \\
\rho(g y, f y, g z), \rho(g x, f y, g z), \rho(g y, f x, g z)\}
\end{array}
$$


for all $x, y, z \in X$ for which the right-hand side is not zero. Further suppose that

(a) $f(X) \subseteq g(X)$,

(b) $\{f, g\}$ is limit coincidentally commuting.

Then $f$ and $g$ have a unique common fixed point.

PROoF. The proof follows by letting $F=\{f\}$, a single-valued mapping in Theorem 4.1.

COROLlary 4.5. Let $X$ be a compact $D$-metric space and let $f: X \rightarrow X$ be a continuous mapping satisfying

$$
\begin{array}{r}
\rho(f x, f y, f z)<\max \{\rho(x, y, z), \rho(f x, f y, z), \rho(x, f x, z), \\
\rho(y, f y, z), \rho(x, f y, z), \rho(y, f x, z)\}
\end{array}
$$

for all $x, y, z \in X$ for which the right-hand side is not zero. Then $f$ has a unique fixed point.

Proof. The conclusion follows by letting $g=I$ in Corollary 4.4, where $I$ is the identity map on $X$.

Note that Corollaries 4.4 and 4.5 include the fixed-point theorems of Dhage [5] and Rhoades [12] for the mappings $f$ and $g$ on a $D$-metric space $X$ characterized by the inequalities

$$
\begin{gathered}
\rho(f x, f y, f z)<\max \{\rho(g x, g y, g z), \rho(g x, f x, g z), \\
\rho(g y, f y, g z), \rho(g x, f y, g z), \rho(g y, f x, g z)\}, \\
\rho(f x, f y, f z)<\max \{\rho(x, y, z), \rho(x, f x, z), \\
\rho(y, f y, z), \rho(x, f y, z), \rho(y, f x, z)\},
\end{gathered}
$$

respectively.

THeOREM 4.6. Let $X$ be a $D$-metric space and let $F: X \rightarrow \mathrm{CB}(X), g: X \rightarrow X$ be two continuous mappings satisfying (4.1). Suppose further that

(a) $F(X) \subseteq g(X)$,

(b) $g(X)$ is compact,

(c) $\{f, g\}$ is coincidentally commuting.

Then $F$ and $g$ have a unique common fixed point $u \in X$ such that $F u=\{u\}=$ gu.

Proof. Let $A=g(X)$. Then $A$ is a compact $D$-metric space and $F$ and $g$ define the maps $F: A \rightarrow \mathrm{CB}(A)$ and $g: A \rightarrow A$. Now, the desired conclusion follows by an application of Theorem 4.1.

Corollary 4.7. Let $X$ be a D-metric space and let $f, g: X \rightarrow X$ be two continuous mappings satisfying (4.8). Further suppose that 
(a) $f(X) \subseteq g(X)$,

(b) $g(X)$ is compact,

(c) $\{f, g\}$ is coincidentally commuting.

Then $f$ and $g$ have a unique common fixed point.

5. Remarks and conclusion. It has been noted in $[6,10]$ that the fixed-point theorems for the limit coincidentally commuting mappings have some nice applications to approximation theory, and therefore it is of interest to discuss the fixed-point theorems for a wide class of coincidentally commuting mappings in a $D$-metric space. The terms "compatible" and " $\delta$-compatible" have been used by Jungck and Rhoades [11] for limit coincidentally commuting and coincidentally commuting mappings, respectively, but our terminologies are natural and more informative than the previous one patterned after [4]. Further we note that a similar study can be made for coincidentally pseudocommuting mappings on a $D$-metric space and analogously for limit coincidentally pseudocommuting mappings. But in order to prove fixed-point theorems for these classes of weakly pseudocommuting mappings, we require a stronger contraction condition for the mappings $F$ and $g$ under consideration:

$$
\begin{aligned}
& \delta^{r}(F x, F y, F z) \\
& \leq \phi\left(\operatorname { m a x } \left\{\rho^{r}(g x, g y, g z), D^{r}(F x, F y, g z), D^{r}(g x, F x, g z),\right.\right. \\
& \left.\left.D^{r}(g y, F y, g z), D^{r}(g x, F y, g z), D^{r}(g y, F x, g z)\right\}\right) .
\end{aligned}
$$

Obviously, condition (5.1) implies condition (3.2) on a $D$-metric space $X$ and hence the fixed-point theorems for weakly pseudocommuting mappings can be obtained very easily with appropriate modifications. Finally, we close this discussion with the following open question.

OPEN QUESTION. Can we prove fixed-point theorems for a class of multivalued mapping $F$ on a $D$-metric space $X$ satisfying the generalized contraction condition

$$
\begin{array}{r}
\kappa(F x, F y, F z) \leq \lambda \max \{\rho(x, y, z), D(F x, F y, z), D(x, F x, z), \\
D(y, F y, z), D(x, F y, z), D(y, F x, z)\}
\end{array}
$$

for all $x, y, z \in X$ and $0 \leq \lambda<1$ ?

ACKNOwledgment. The authors are thankful to Professor B. E. Rhoades (USA) for giving some useful suggestions for the improvement of this paper.

\section{REFERENCES}

[1] B. C. Dhage, A study of some fixed point theorem, Ph.D. thesis, Marathwada University, Aurangabad, India, 1984.

[2] _ Generalised metric spaces and mappings with fixed point, Bull. Calcutta Math. Soc. 84 (1992), no. 4, 329-336. 

, Generalized D-metric spaces and multi-valued contraction mappings, An. Ştiinț. Univ. Al. I. Cuza Iaşi. Mat. (N.S.) 44 (1998), no. 1, 179-200.

[4] _ A common fixed point principle in D-metric spaces, Bull. Calcutta Math. Soc. 91 (1999), no. 6, 475-480.

[5] _ On common fixed points of pairs of coincidentally commuting mappings in D-metric spaces, Indian J. Pure Appl. Math. 30 (1999), no. 4, 395-406.

[6] _ Applications of some fixed point theorems in approximation theory, Math. Sci. Res. Hot-Line 4 (2000), no. 9, 63-69.

[7] _ A common fixed point theorem for a pair of limit coincidentally commuting mappings in D-metric spaces, Math. Sci. Res. Hot-Line 4 (2000), no. 2, 4555.

[8] _ _ Generalized metric spaces and topological structure. I, An. Ştiinț. Univ. Al. I. Cuza Iași. Mat. (N.S.) 46 (2000), no. 1, 3-24.

[9] __ On some fixed point theorems for contractive mapping in D-metric spaces, An. Ştiinț. Univ. Al. I. Cuza Iaşi. Mat. (N.S.) 46 (2000), no. 1, 31-38.

[10] _ A result in best approximation theory, Indian J. Pure Appl. Math. 32 (2001), no. 8, 1209-1216.

[11] G. Jungck and B. E. Rhoades, Fixed points for set valued functions without continuity, Indian J. Pure Appl. Math. 29 (1998), no. 3, 227-238.

[12] B. E. Rhoades, A fixed point theorem for generalized metric spaces, Int. J. Math. Math. Sci. 19 (1996), no. 3, 457-460.

B. C. Dhage: Kasubai, Gurukul Colony, Ahmedpur 413515, Maharashtra, India

E-mail address: bcd20012001@yahoo.co.in

A. Jennifer Asha: Ramanujan Institute for Advanced Study in Mathematics, University of Madras, Chennai 600005, India

S. M. Kang: Department of Mathematics and Research Institute of Natural Science, Gyeongsang National University, Chinju 660-701, Korea

E-mail address: smkang@nongae.gsnu.ac.kr 


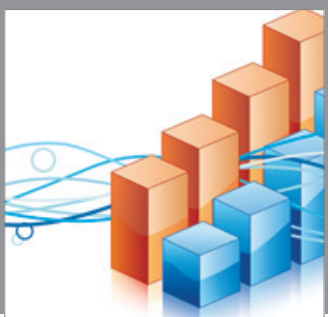

Advances in

Operations Research

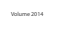

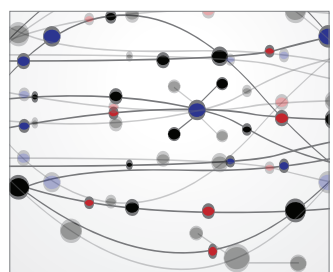

\section{The Scientific} World Journal
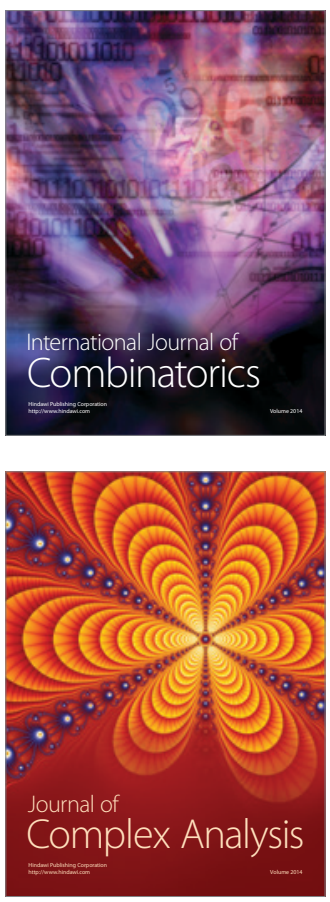

International Journal of

Mathematics and

Mathematical

Sciences
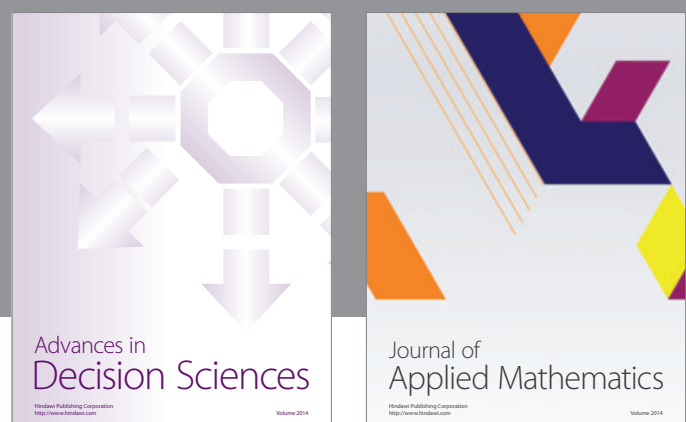

Journal of

Applied Mathematics
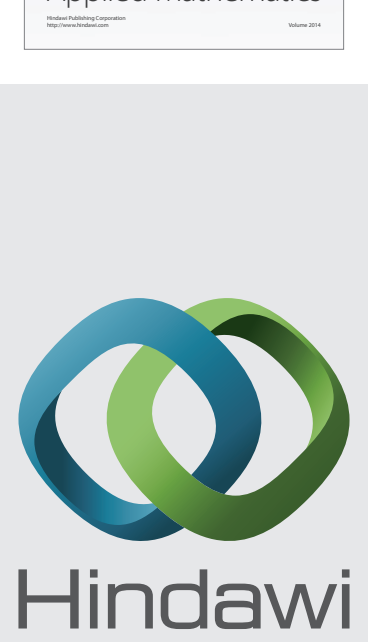

Submit your manuscripts at http://www.hindawi.com
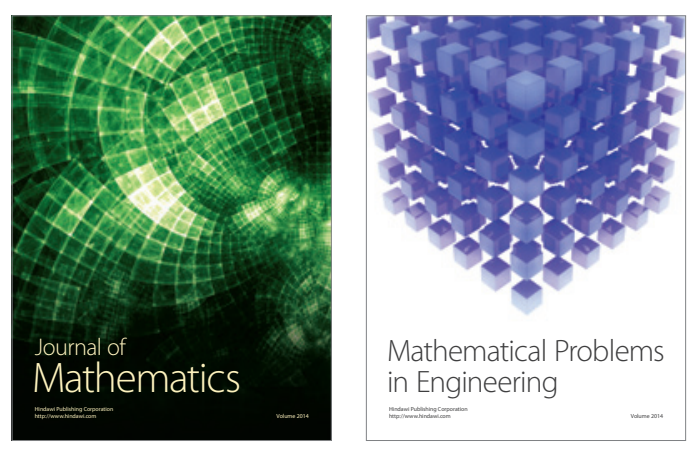

Mathematical Problems in Engineering
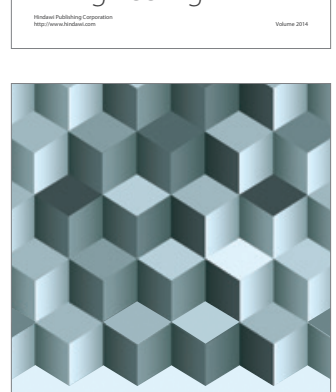

Journal of

Function Spaces
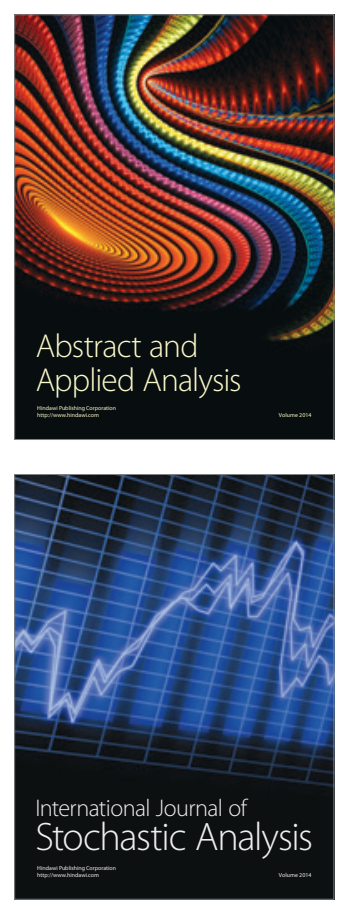

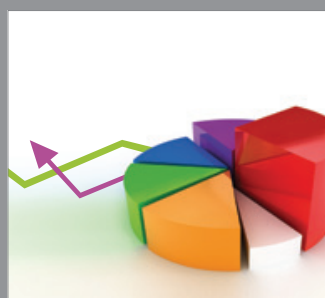

ournal of

Probability and Statistics

Promensencen
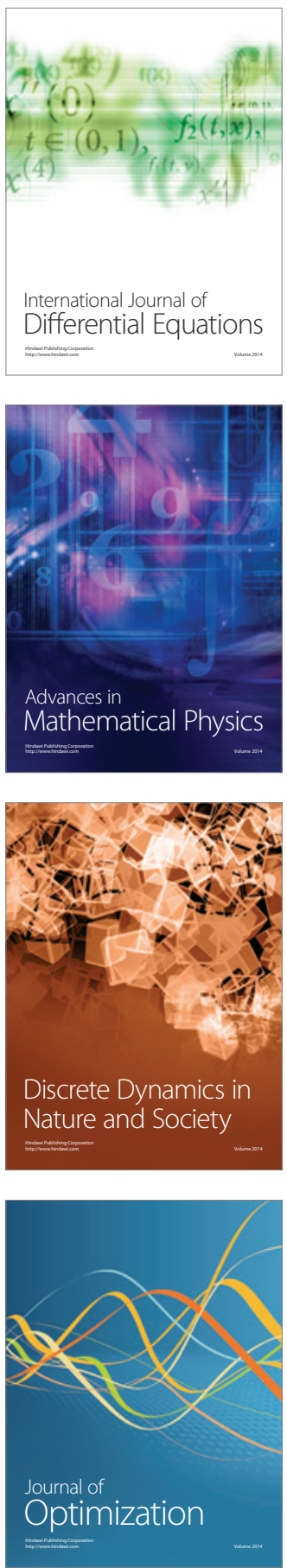